\title{
Resection of Foramen Magnum Meningioma through Modified Far Lateral Approach: Surgical Principles and Technical Nuances
}

\author{
Vinayak Narayan ${ }^{1}$ Fareed Jumah ${ }^{1}$ Anil Nanda ${ }^{1}$ \\ 1 Department of Neurosurgery, Rutgers Robert Wood Johnson Medical \\ School and University Hospital, New Brunswick, New Jersey, United States \\ Address for correspondence Anil Nanda, MD, MPH, FACS, \\ Department of Neurosurgery, Rutgers Robert Wood Johnson Medical \\ J Neurol Surg B 2021;82(suppl S1):S22-S24. \\ School, New Brunswick, Rutgers New Jersey Medical School, Newark, \\ NJ 08901, United States (e-mail: an651@rwjms.rutgers.edu).
}

\begin{abstract}
Objectives Safe maximal resection is the basic principle of cranial base surgery and the grade of resection is an important factor influencing the prognostic outcome. This operative video highlights the surgical principles and technical nuances in the microsurgical resection of foramen magnum meningioma (FMM).

Case Description The surgery was performed in a 45-year-old lady who presented with hoarseness of voice and spastic quadriparesis (grade 4/5). On imaging, FMM with mass effect on brainstem and spinal cord was identified. The tumor was gross totally resected through modified far lateral approach with minimal occipital condyle drilling. This video demonstrates the surgical techniques of tumor resection including early devascularization, operating in the arachnoid plane to dissect the neurovascular structures, piecemeal decompression, sharp dissection to separate tumor from lower cranial nerves (LCN), identifying the brainstem veins, and resecting the lesion from tumor-brainstem interface. Postoperatively, she had significant neurological improvement and the magnetic resonance imaging revealed excellent radiological outcome (- Figs. 1 and $\mathbf{2}$ ).

Conclusion The surgery of FMM is challenging due to the deep surgical corridor, critical location, close proximity with various neurovascular structures, firm consistency, and high

Keywords

- meningioma

- modified far lateral

- foramen magnum

- resection

- approach vascularity of the tumor. The modified far lateral approach by preserving the occipital condyle may prevent the postoperative incidence of craniovertebral junction instability. The key operative principles to achieve the best surgical outcome include careful dissection along the arachnoid plane, gentle handling of cranial nerves, veins, and perforator vessels, avoidance of traction on brainstem and spinal cord, intraoperative neurophysiological monitoring, proper hemostasis, and meticulous dural closure.

The link to the video can be found at: https://youtu.be/1qvAeUmNIUw.
\end{abstract}

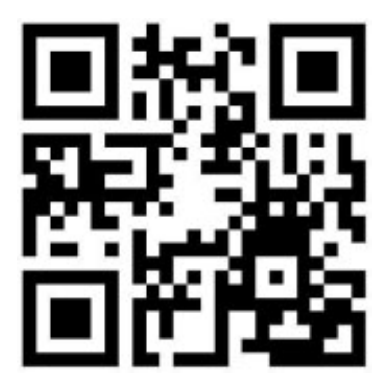

received

February 28, 2019 accepted after revision March 8, 2020

published online

November 23, 2020

\section{Conflict of Interest \\ None declared.}

www.thieme.com/skullbasevideos

www.thieme.com/jnlsbvideos

DOI https://doi.org/ 10.1055/s-0040-1714405. ISSN 2193-6331.

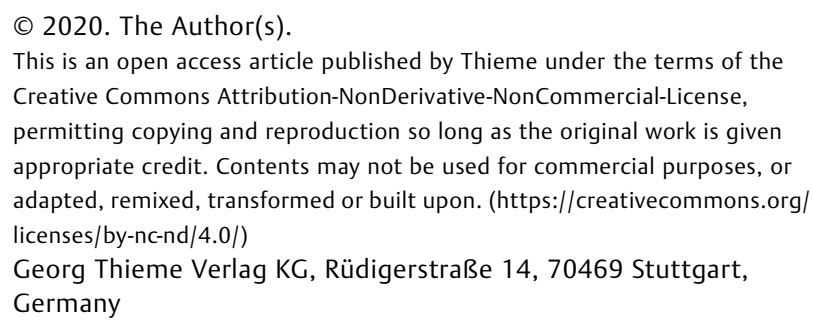



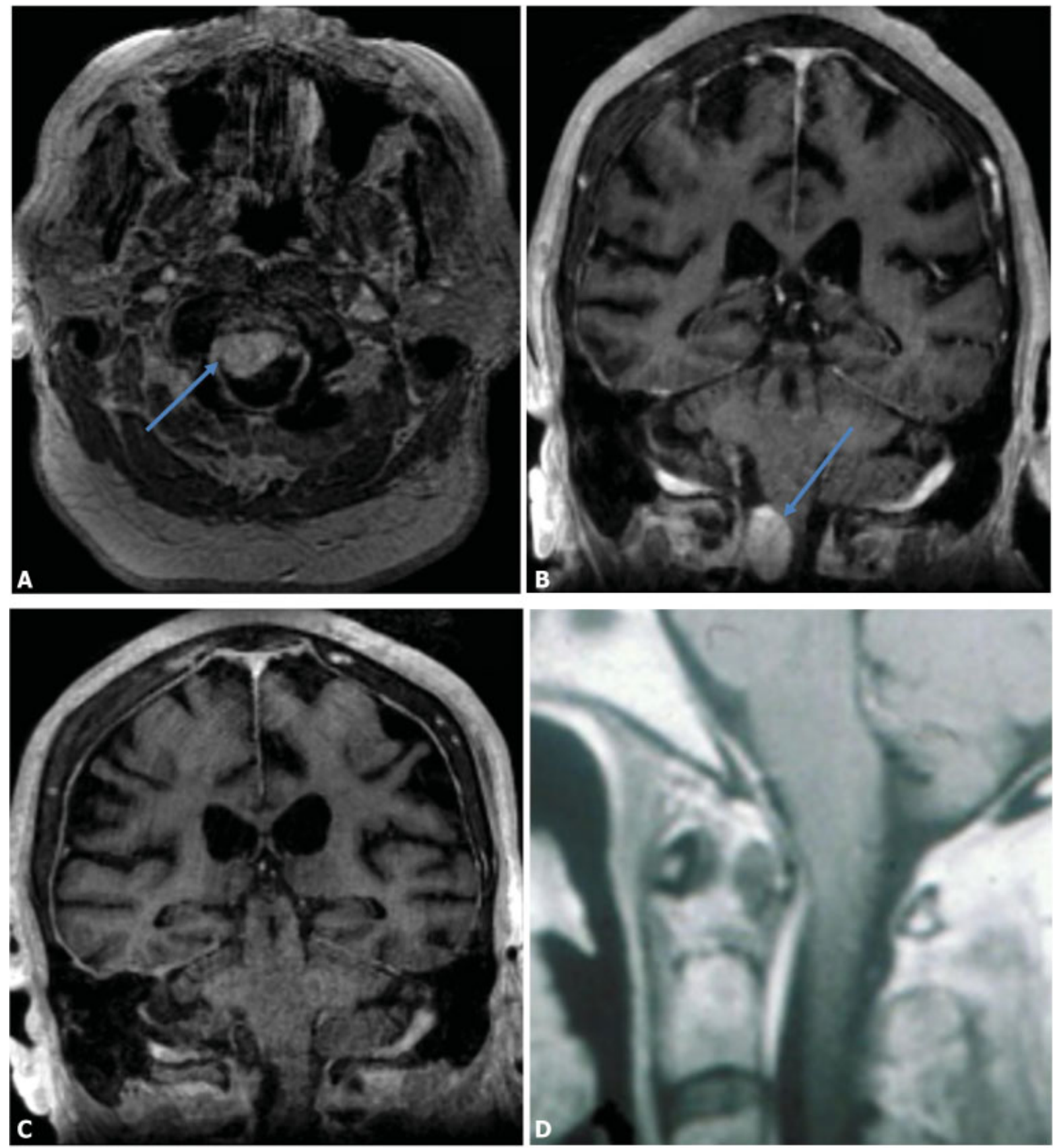

Fig. 1 Radiology panel showing the pre and postoperative images of foramen magnum meningioma (FMM) resection. (A) Preoperative axial MR image showing FMM (blue arrow). (B) Preoperative coronal MR image showing FMM (blue arrow). (C) Postoperative coronal MR image showing gross total resection of tumor. (D) Postoperative sagittal MR image showing gross total resection of tumor. MR, magnetic resonance. 

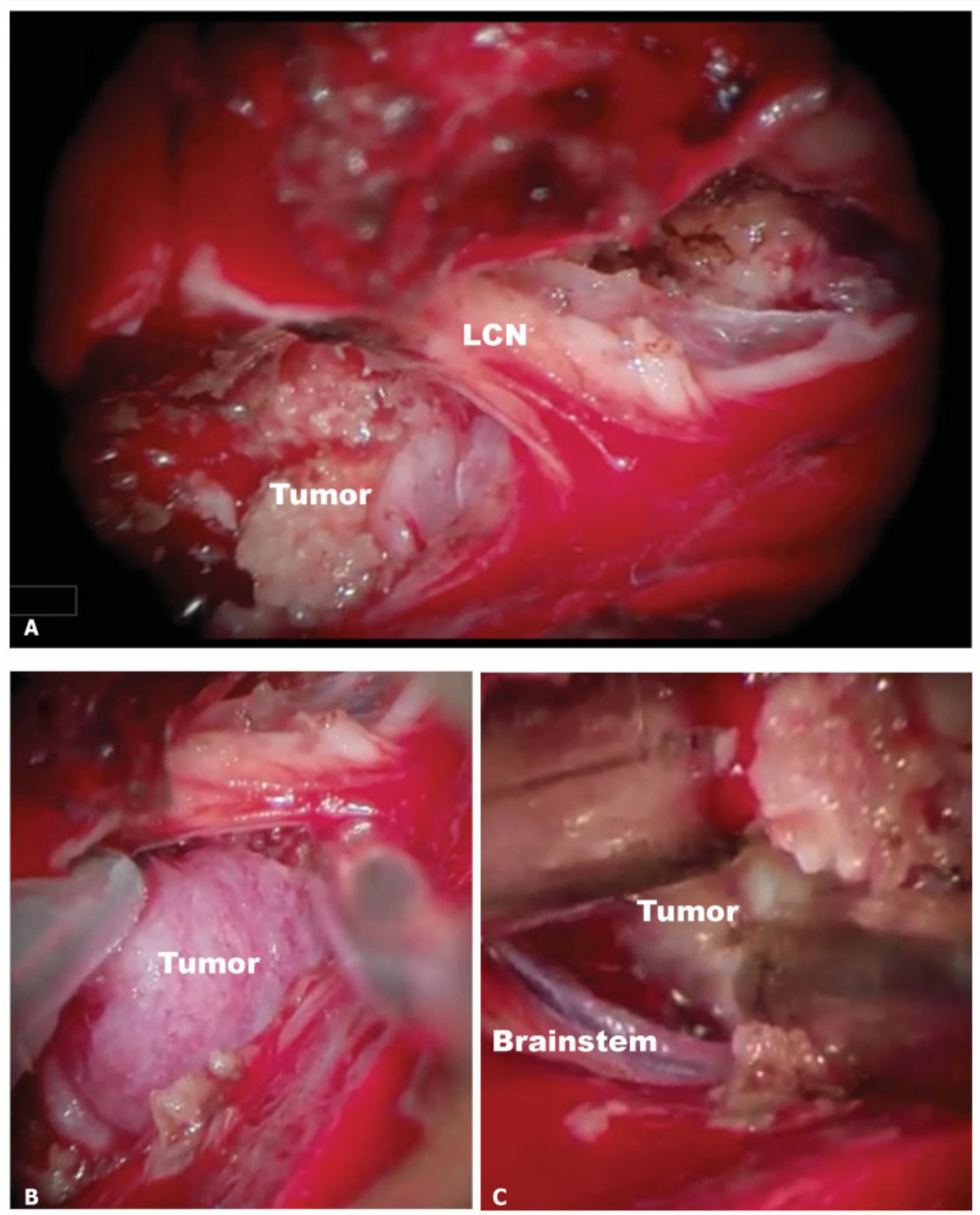

Fig. 2 Panel showing the intraoperative images of foramen magnum meningioma (FMM) resection. (A) Tumor in relation to the lower cranial nerves (LCN). (B) Mobilization of tumor from the surrounding anatomical structures. (C) Separation of tumor from the brainstem surface. 